Relations industrielles

Industrial Relations

\title{
La propriété dans la société nord-américaine
}

\section{Edward Duff}

Volume 15, numéro 1, janvier 1960

URI : https://id.erudit.org/iderudit/1022070ar

DOI : https://doi.org/10.7202/1022070ar

Aller au sommaire du numéro

\section{Éditeur(s)}

Département des relations industrielles de l'Université Laval

ISSN

0034-379X (imprimé)

1703-8138 (numérique)

Découvrir la revue

Citer cet article

Duff, E. (1960). La propriété dans la société nord-américaine. Relations industrielles / Industrial Relations, 15(1), 54-95.

https://doi.org/10.7202/1022070ar
Résumé de l'article

L'auteur confronte ici la doctrine traditionnelle de la propriété privée avec le contexte économique nord-américain.
Tous droits réservés Département des relations industrielles de l’Université Laval, 1960
Ce document est protégé par la loi sur le droit d'auteur. L’utilisation des services d'Érudit (y compris la reproduction) est assujettie à sa politique d'utilisation que vous pouvez consulter en ligne.

https://apropos.erudit.org/fr/usagers/politique-dutilisation/ 


\title{
La propriété dans la société nord-américaine
}

\section{Edward Duff}

\begin{abstract}
L'auteur confronte ici la doctrine traditionnelle de la propriété privée avec le contexte économique nord-américain.
\end{abstract}

Dans la pensée sociale du catholicisme, la notion de propriété s'est toujours trouvée liée au problème de la dispersion de la puissance économique, et de la nécessité d'assurer le développement de l'individu. On attribue généralement à la propriété une fonction triple: 1) en assurant l'indépendance économique de l'individu, elle protège ses libertés; 2) en garantissant la possession incontestée de certains biens de valeur, elle offre à l'individu, à sa famille et à ses héritiers une sécurité en période de crise aussi bien que pour leur vieillesse; 3) en développant l'esprit créateur et la personnalité, du fait qu'elle donne naissance à ce que nous appelons justement le sens de la propriété.

Tels sont les arguments traditionnels de notre plaidoirie en faveur d'une très vaste répartition de la propriété privée. ${ }^{1}$ C'est avec eux que nous étayons, dans notre programme d'éthique sociale, notre réfutation du communisme d'une part, du «laissez faire» capitaliste de l'autre. Il faut souligner que ces arguments n'expriment pas seulement la conviction des sociologues catholiques. Formulés dès le début de la Guerre d'Indépendance, ils allaient devenir partie intégrante de la tradition politique américaine. Ce fut Thomas Jefferson qui leur donna une forme concrète en affirmant que l'expérience tentée aux Etats-Unis serait condamnée le jour où la nation cesserait d'être une société de petits possédants, sans milliardaires ni déshérités. Si le petit fermier et l'artisan indépendant devraient céder la pla$\propto$ à une masse ide travailleurs sans propriété aucune, la démocratie ne tarderait guère à

DUFF, EDward, s.j., directeur de la revue Social Order, Institute of Social Order, St. Louis, Missouri.

(1) Dans son analyse de la nature individuelle et sociale de l'homme, Messmer trouve certes 12 motifs du droit à la propriété privée, mais ce ne sont que des développements ou perfectionnements des trois raisons indiquées plus haut. Voir son Social Ethics (Herder, St. Louis, 1952), p. 787-790. Le dominicain anglais Drostan Maclaren résume ainsi la doctrine de S. Thomas: \& L'homme 
succomber. Jefferson insistait fréquemment sur cette menace, employant des images frappantes qui, jusqu'à ces dernières années, trouvaient encore une vaste audience.

Cependant, dans ce domaine, nous nous heurtons à un certain nombre de faits troublants.

De nos jours, le régime de la propriété privée très largement réparti, tel qu'il est préconisé dans nos manuels de morale, c'est-à-dire sous la forme de propriété immobilière ou de moyens de production, n'existe nulle part dans le monde occidental. Peut-être n'a-t-il jamais existé, du moins poussé au point que supposait notre argumentation. En effet, il semble qu'on lui ait attribué une importance excessive, d'abord à la suite des affirmations des physiocrates français du XVIIIe siècle, puis de certaines tendances romaines qui commencèrent à se manifester sous Léon XIII. Quoi qu'il en soit, l'idéal d'une société dominée par le petit fermier (le «paysan» des pays d'Europe Orientale) et par l'artisan indépendant n'a plus aujourd'hui qu'une signifcation des plus restreintes, bien que certains orateurs politiques croient toujours utile de l'invoquer.

Pourtant, - à notre étonnement, peut-être, - les conséquences logiques prédites par nos manuels ne se sont guère manifestées. L'ouvrier américain ne se considère nullement comme réduit à l'esclavage par son patron. De plus, les brillants succès de la science soviétique semblent indiquer qu'il existe, dans le domaine de la création, des stimulants autres que ceux de la propriété. Quant à la sécurité économique, l'Occident n'a encore jamais connu une période où l'on voit aussi peu de gens mourir de faim ou de maladie, faute de pouvoir accéder aux produits alimentaires ou aux soins médicaux.

Comment expliquer cette anomalie? Notre analyse en faveur de la propriété privée était-elle incomplète, mal fondée sur un raisonnement empirique, comme le fut, par exemple, notre condamnation morale du droit de vote des femmes? D’autres influences se seraientelles substituées aux bienfaits de la propriété, de nouveaux facteurs auraient-ils réduit à néant les maux attribués par une longue tradition

devant conserver son essence, il doit avoir le droit de posséder des moyens d'existence. L'homme devant procréer, il doit être en mesure d'utiliser des biens matériels afin de protéger, nourrir et élever ses enfants. L'homme doit vivre selon sa nature rationnelle, étant créé pour connaître et aimer Dieu, pour vivre en société, avec ses semblables. Ce qui exige pour le moins le droit général à la propriété \$. Voir Private Property and the Natural Law, Aquinas Papers no 8, Oxford, 1948, p. 18. 
à son déclin? Faut-il élargir notre notion de la propriété privée, du jus procurandi et dispensandi, ou tout au moins la débarrasser d'un contexte qui la relie trop étroitement à la propriété immobilière?

Autant de questions dont l'importance dépasse certainement le cadre de la simple actualité.

\section{La répartition de la propriété}

Pour commencer, il faut évidemment déterminer avec un maximum de précision le tableau présent de la situation aux Etats-Unis, dans une société qui, sans aucune honte, se définit elle-même comme capitaliste, dans un pays où il n'existe pas de parti socialiste viable (ni même visible).

Il serait sans doute facile, - et erroné - de justifier l'affirmation d'après laquelle l'Amérique, sans être une «nation de petits boutiquiers » comme disait Napoléon en parlant des Anglais, serait caractérisée par l'extension et la dispersion de la propriété privée.

«On compte aux Etats-Unis 4,3 millions d'entreprises auxquelles il faut ajouter 3,3 millions de fermes à exploitation commerciale et une très forte proportion des 550,000 membres des professions libérales, - médecins, dentistes, avocats, architectes, conseillers divers ayant un «cabinet». Au total, quelque 8 millions de «firmes» que l'on peut considérer comme un énorme groupement d'équipes économiques ». "

On pourrait également noter qu'on comptait aux Etats-Unis, en 1950, environ 46 millions d'unités d'habitation: maisons individuelles, immeubles de rapport, grands ensembles. L'unité moyenne abritait 3,5 personnes; quatre unités sur cinq étaient des maisons habitées par une seule famille; et $55 \%$ de l'espace de logement appartenaient en propre aux occupants. Au dernier recensement décennal, on comptait 42,827,000 habitations occupées par le propriétaire. Si l'on attribue à chacune de ces habitations la valeur relativement modeste de $\$ 10,000$., on arrive à cette constatation que la fortune immobilière répartie entre les Américains représente 428 milliards de dollars. De même,

(2) Economic Growth in the United States: Its Past and Future. Publié par le Service d'Ftudes du Comité pour le Développement Economique, New-York, 1958, p. 20. 
les 60 millions d'automobiles des Etats-Unis constituent une masse concrète et impressionnante de propriété privée. (Reste à savoir dans quelle mesure cette propriété est libre de dettes. Plus de $50 \%$ des Américains dépensant leur propre argent ne possèdent même pas $\$ 500$. en espèces.).

On pourrait encore souligner, - et on le fait fréquemment l'immense dispersion des titres de bourse. Pour ne citer qu'un exemple: les actions de la Telephone and Telegraph Company sont réparties entre plus de $1,600,000$ personnes. Sur les 25 principales sociétés industrielles des Etats-Unis, 19 comptent plus d'actionnaires que de salariés. $^{3}$ Cette évolution a donné naissance à un terme nouveau, le «Capitalisme populaire», formule inventée par le Conseil de la Publicité et figurant dans le texte d'un panneau que l'Agence d'Information des Etats-Unis diffusa dans le monde entier.

L'économiste Marcus Nadler utilisa, lui aussi, ce terme dans une brochure à gros tirage. "L'économie américaine, affirmait-il, se transforme rapidement dans le sens de ce qu'on pourrait appeler un \& capitalisme populaire». Dans ce régime, la possession des moyens de production, notamment au point de vue industriel, se généralise de phus en plus parmi les personnes disposant de revenus moyens ou modestes, soit directement, soit indirectement, par l'intermédiaire d'organismes mutuels qui gèrent leurs économies ». ${ }^{4}$

Nadler constate que le nombre des détenteurs de titres américains a passé de 6,5 millions en 1952 à 8,6 millions en 1955 . Il note que phis de $50 \%$ de ces titres appartiennent à des familles dont le revenu ammel se situe entre $\$ 3,000$, et $\$ 5,000$. Il se réjouit de la progression surprenante des fonds investis dans des organismes mutuels ot qui, à la fin de l'année 1955, dépassaient 9 milliards de dollars. Ce chiffre, estime-t-il, prouve que les petits épargnants, en général des salariés, recourent de plus en plus aux professionnels du marché qu'ils chargent du soin de composer et de gérer leur portefeuille. Il parle également des 16,4 millions de comptes dans des Caisses d'Epargne et Sociétés de Crédit, d'un montant global de près de 38 milliards de dollars à la fin 1954, des 20,9 millions de comptes, d'un moztant de 31 milliards, dans les Banques d'Epargne Mutuelle. Il pourrait ajou-

(3) B. Brewster Jennings. A Richer Life in a Poorer World. Publié par la Socony Mobil Oil Company, p. 6.

(4) People's Capitalism, New Yerk. Publié par la Hanover Bank, 1956, p. 5. 
ter que de nombreuses entreprises ont mis au point des programmes d'achats boursiers pour leurs employés.

Or, cette argumentation est forcée; voulant trop prouver, elle brosse un tableau qui ne constitue qu'une demi-vérité. Tout d'abord, les Américains possédant serait-ce un seul titre de bourse forment une minorité: moins de 1 sur 10. Fait plus important, le fonctionnement des grandes sociétés réduit les détenteurs des titres à un rang difficilement conciliable avec notre notion traditionnelle de la propriété.

L'instrument à la fois caractéristique et dominant de la production américaine est l'entreprise géante: les 500 plus grosses sociétés dirigent à elles seules les deux tiers de notre économie. Dans le mécanisme légal qui est à la base de leur organisation, la propriété ne coïnoide pas nécessairement avec le contrôle effectif. La société-type représente une concentration de capitaux provenant d'un grand nombre de personnes et destinée à financer la production commerciale de marchandises. Le processus de production est généralement confié à des directeurs, nommés par et responsables devant les membres du Conseil d'Administration. Ceux-ci, à leur tour, sont légalement responsables devant les actionnaires; il arrive, toutefois, qu'ils ne sont responsables envers personne. Ceci parce que l'actionnaire s'en désintéresse, qu'en plus, il ne possède pas les capacités nécessaires pour exercer, même de la façon la plus vague, le droit de surveillance directe que lui assure, légalement, son égalité avec les autres co-propriétaires. En somme, son titre ne constitue guère qu'un droit à sa part des profits. Fait plus grave, la plupart des actionnaires ne tiennent nullement à être des «propriétaires» dans le sens réel du terme. Ce sont, d'après la définition de J-A. Livingston, rédacteur financier du Philadelphia Bulletin, «des gens qui ont fait un placement, mais qui ne veulent pas être dérangés, - sauf pour encaisser des dividendes $\$ .5$

Le petit capitaliste moyen a acquis tel titre afin de gagner de l'argent, soit grâce aux dividendes, soit grâce à une montée des cours; peut-être aussi pour se protéger contre une éventuelle dévaluation. Lorsqu'il est mécontent de la gestion des directeurs ou de la politique suivie par le Conseil d'Administration, il ne cherchera qu'exceptionnellement à exercer ses droits de co-propriétaire; il liquidera ses titres, tout simplement. De toute manière, il ne peut guère faire autrement.

(5) The American Stockholder, Lippincott, Philadelphie, 1958, p. 38. 
Un enquête portant sur 3,7 milliards d'actions, - plus de $75 \%$ du total (estimé) des titres offerts au public — a révélé que:

- Les deux tiers environ des portefeuilles constitués par ces titres comprennent moins de 100 actions. Quant aux détenteurs individuels, le portefeuille moyen est nettement au-dessous de ce chiffre ». ${ }^{\text {" }}$

Or, avec un paquet de 100 titres, l'actionnaire qui voudrait exercer une influence quelconque sur la direction de la société se trouve réduit à une impuissance telle que seul un exhibitionniste forcené songerait à s'inspirer de l'égalité de tous les co-propriétaires pour consommer le déjeuner gratuit offert à l'occasion de l'assemblée générale et proclamer ensuite son désaccord. De plus, l'actionnaire moyen, homme de moyens modestes, n'aura guère l'ambition insensée de prétendre à un droit de regard sur la société. En pratique, il n'aurait aucune possibilité d'exercer le moindre contrôle: l'énorme dispersion des titres et le fait que les directeurs gardent la haute main sur les votes par procuration constituent un obstacle fondamental et presque insurmontable pour les actionnaires qui voudraient s'organiser afin de livrer bataille à un Conseil d'Administration solidement retranché. Le revenu moyen de l'actionnaire américain est de $\$ 6,300$. par an, ce qui équivaut au salaire d'un ouvrier qualifié. Sans aucun doute, ses achats en bourse démontrent sa foi en l'expansion continue de l'industrie américaine, tout comme les efforts entrepris pour stimuler son intérêt aux opérations boursières montrent que les milieux d'affaires ont découvert en lui une nouvelle source de capitaux. Une importante étude d'agents de change a installé un bureau au carrefour de la Gare Centrale de New-York, afin de drainer vers ses guichets les opérations des voyageurs; et le Stock Exchange de New-York mène campagne pour ce qui revient pratiquement à un achat à tempérament de valeurs mobilières.

Pourtant, aucun de ces millions de propriétaires d'actions ne saurait se prévaloir de son portefeuille pour exercer un contrôle réel. Fait dont la Du Pont de Nemours a, par inadvertance, fourni la démonstration dans une brochure qui proclame fièrement: «Aujourd'hui, les épargnants représentent une coupe transversale de la nation américaine 3. Sur les photographies accompagnant cette affirmation, on voyait un joueur de golf professionnel, un fonctionnaire, un étudiant, une ménagère, un directeur du personnel, une femme d'affaires et un

(6) Lewis H. Kommex, Share Ownership in the United States, Washington, The Brookings Institution, 1952, p. 126. 
professeur, censés refléter la composition sociale de près de 200,000 actionnaires. $^{7}$ Une autre photo, à la page précédente, montrait l'assemblée annuelle des actionnaires, - en tout, quelque 800 personnes.

Dans une analyse féroce de l'allégation selon laquelle la grande dispersion de la propriété boursière entraînera un changement aux leviers de commande de l'économie américaine, Victor Perlo écrivit récermment:

* Vous aurez beau chercher, vous ne trouverez pas un seul représentant authentique des petits actionnaires parmi les dirigeants des grosses sociétés. Et cela que l'on définisse le représentant comme ayant une occupation similaire à celles du petit actionnaire-type (commerçant, fonctionnaire, salarié), ou comme un délégué d'un groupement de l'une de ces catégories ${ }^{8}{ }^{8}$

Propiété anonyme et direction autonome apparaissent ainsi comme les caractéristiques des entreprises géantes d'aujourd'hui. ${ }^{\circ}$

Quelle est la domination réelle qu'exerce cette forme de propriété, - l'entreprise géante - sur l'économie américaine? Le professeur Adelman a calculé que 135 sociétés se partagent $45 \%$ de l'actif industriel (c'est-à-dire ni agricole ni financier) des Etats-Unis ou, en d'autres termes, presque le quart de la capacité industrielle mondiale. ${ }^{10}$ Cette estimation concorde, à peu de chose près, avec un rapport publié plus tôt par la Commission Fódérale du Commerce et intitulé la Concentration des Moyens de Production. Dans tel marché, la domination réelle revient à un nombre extrêmement réduit de sociśtés; sowvent,

(7) This is Dupont: L'histoire de la Vie dans une Grande Entreprise. Wilmington (Del.) E.I. Du Pont de Nemours et Co., 1957, p. 26.

(8) People's Capitalism and Stock-Ownership, American Economic Review, juin 1958 , p. 348.

(9) Il y a vingt-cinq ans, Berle et Means démontrèrent que \& dans le système de la société par actions, le \&propriétaire» des biens industriels doit se contenter d'un simple symbole de possession, tandis que le pouvoir, la responsabilité, et même la substarece, autrefois parties intégrantes de la possession, se trouvent transférés à un groupe autonøme qui détient tous les rouages \$. Voir Sociétés par Actions d'aujourd'hui, et Propriété Privée, MacMillan, New-York, p. 68. L'été dernier, le Père James L. Vizzard, de la Compagnie de Jésus, après avoir exposé que le propriétaire immobilier \& avait quelque chose où il peut s'accrocher, pour laquelle il peut lutter $»$, posa aux membres de l'American Life Association la question suivante: \&Où voulez-vous qu'un actionmaire de la General Motors ou de la Telephone and Telegraph Company aille prendre position? Devant une agence de vente de voikures, ou devant un poteau télégraphique? ». Work, août 1958.

(10) The Measurement of Industrial Concentration, Review of Economics and Statistics, Bøston, 1952, p. 42-3. 
elles ne sont que quatre à contrôler pratiquement telle ou telle branche d'activité. Pour citer le professeur John K. Galbraith:

- Dans la production des voitures, machines agricoles, pneus, cigarettes, aluminium, alcool, conserves de viande, cuivre et outillage de bureau, les trois firmes les plus importantes réalisaient en 1947 les deux tiers du chiffre d'affaires total. Dans l'industrie de l'acier, du verre, des produits chimiques et des produits laitiers, les six entreprises principales représentaient encore les deux tiers du chiffre d'affaires. La concentration est à peu près similaire dans bon nombre d'industries moins importantes ou dérivées. Dans d'autres domaines encore, l'essence, le ciment, les engrais, la distribution du lait, - les manchés, nécessairement limités régionalement ou localement, sont partagés entre un nombre tout aussi restreint de distributeurs ». ${ }^{11}$

Peut-être un tableau indiquant la part des quatre plus grosses entreprises dans certaines branches industrielles traduira-t-il mieux cette situation. Nous avons emprunté les chiffres au rapport publié par la sous-commission d'enquête du Congrès sur les trusts et monopoles, sous le titre «La Concentration dans l'Industrie Américaine \$:

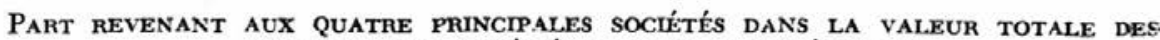
MARCHANDISES EXPÉDIÉES, ET LA MAIN-D'OEUVRE

(Sélection de l'Industrie Américaine, 1954)

Industries fortement concentrées

Aluminium brut

Industrie du plâtre

Equipement téléphonique et télégraphique

Machines à vapeur et turbines

Savon et glycérine.

Cigarottes

Fibres synthétiques

Ferblanterie

Pneus, chambres à air.

Transformateurs.

Voitures et pièces détachées

Machines à calculer.

Tracteurs

Machines à laver

Raffineries de sucre

Hauts fourneaux

Tubes électroniques

Appareils photographiques

Aviation (moteurs)

\section{Marchandises expédiées (en \%) \\ Personnel employé (en \%)}

$\begin{array}{rr}100 & 100 \\ 90 & 86 \\ 89 & 88 \\ 87 & 86 \\ 85 & 71 \\ 82 & 75 \\ 80 & 73 \\ 80 & 78 \\ 79 & 80 \\ 78 & 71 \\ 75 & 72 \\ 74 & 72 \\ 73 & 72 \\ 68 & 60 \\ 67 & 69 \\ 65 & 63 \\ 64 & 63 \\ 63 & 61 \\ 62 & 51\end{array}$

(11) Amerioan Capitalism, Houghton Mifflin, Boston, 1952, p. 42-3. 
Cokeries.

$\begin{array}{ll}58 & 53 \\ 54 & 53 \\ 50 & 47\end{array}$

Scieries et laminoirs.

53

Moteurs et génératrices.

Industries à faible concentration

Confiserie.

Papeterie

Outillage de construction et minier

Machines-outiis..

Bonneterie (coton)

Journaux.

Tanneries, cuirs finis..

Equipement pour élevage de poules.

Joints et garnitures.

Cartonnages

Matrices métalliques.

Confection pour hommes.

Imprimerie commerciale.

Ameublement (bois)

Ateliers de machines

Matières plastiques

Cependant, même ces chiffres ne traduisent pas entièrement l'énorme puissance des grandes sociétés dans l'économie américaine. Citons un exposé récent d'Adolf A. Berle (Jeune), co-auteur, avec Gardiner C. Means, de l'enquête retentissante publiée en 1932 sous le titre \& La société par actions moderne, et la propriété privée »:

«La très grosse société a parfaitement le pouvoir de prendre des décisions et, même, d'accomplir des opérations qui dépassent le cadre de ce qui lui appartient en propre. Sa puissance s'étend bien au-delà de ses titres, bien que nous ne possédions encore aucune estimation statistique quant à l'importance de ce pouvoir périphérique ». ${ }^{12}$

M. Berle estime, par exemple, que les capitaux investis en garages et ateliers de réparation concessionnaires des grandes marques de voitures représentent environ 3 milliards de dollars. Un total impressionnant, réparti entre des propriétaires théoriquement indépendants, - théoriquement, car les contrats, opérations et, dans une large mesure, les prix pratiqués par ces concessionnaires sont déterminés par les compagnies dont ils vendent les voitures. D'un autre côté, la General Motors par exemple a quelque 21,000 fournisseurs auxquels elle versa, en 1955, environ 6 milliards de dollars, somme fantastique qui

(12) Le Capitalisme américain et la conscience du Roi; le néo-capitalisme aux Etats-Unis. Armand Colin, 1957. 
lui confère une énorme puissance sur le marché des fournitures. Citons encore, parmi les exemples les plus frappants, le propriétaire d'une pompe à essence vis-à-vis de la société pétrolière qui lui fournit ses produits. Or, la seule Socony Mobil Oil Company et ses filiales américaines approvisionnent 2,700 distributeurs indépendants et 34,000 vendeurs également «indépendants ».

Au cours de ces dernières années, la tendance à la concentration ne s'est pas développée outre mesure, dans l'industrie américaine. En revanche, un nouveau phénomène risque de réduire encore le nombre des individus qui contrôlent effectivement l'économie du pays. Il s'agit de l'accroissement des fonds privés de retraite.

\section{Les effets des fonds de retraite}

Le système des retraites versées par l'entreprise reçut une impulsion puissante au cours de la dernière guerre, grâce à la politique de Washington en matière de salaires. Les salaires étant officiellement stabilisés et les augmentations interdites, les employeurs, mis en compétition par la pénurie de main-d'oeuvre, se voyaient forcés, afin d'attirer les ouvriers, d'offrir des suppléments non inflationnaires, sous forme de retraite ou d'assurance-maladie. ${ }^{13}$ Sous la pression des syndicats, et avec l'aocord du gouvernement, cette pratique se généralisa très vite dans les industries à production massive. Le syndicat des Mineurs, par exemple, négocia en 1946 un contrat collectif aux termes duquel les employeurs acceptaient de verser, à un fonds de retraite, une somme de 5 cents (aujourd'hui, 40 cents) par tonne de charbon extraite. L'inclusion d'un programme de retraites dans les contrats collectifs devenait ainsi légale et, en pratique, obligatoire. Puis, dès le début de la Guerre de Corée, le gouvernement imposa de nouveau la stabilisation absolue des salaires. Si bien que même des entreprises dont le personnel n'était pas syndiqué se trouvèrent forcées d'offrir des retraites si elles voulaient garder leurs ouvriers.

A la fin de l'année 1956, le total de ces fonds de retraite privés atteignait quelque 30 milliards de dollars, somme supérieure aux réserves gouvernementales $(22,5$ milliards) pour le programme officiel

(13) Voir Paul Harbrecht, s.j., \& Property in Transition \$, Social Order, février 1958. Les travaux du Père Harbrecht dans ce domaine seront bientôt publiés par le Twentieth Century Funds sous le titre Pension Funds and Economic Power. 
d'assurance-vieillesse. Or, plus de $50 \%$ de l'actif de ces fonds privés, - 16,6 milliards de dollars - sont groupés dans des comptes qui ne sont pas gérés par les compagnies d'assurances. Aujound'hui, ces fonds s'accroissent plus rapidement que toute autre concentration de capitaux: dans la seule année 1956, ils ont augmenté de 2,4 milliards. Et ils continueront inévitablement à s'accroître, à mesure que s'accrôitra la capacité de travail de la nation, qu'une fraction toujours plus importante du salariat accédera à ces avantages, qu'un nombre toujours plus grand des programmes privés disposeront d'une base financière suffisante pour constituer des réserves correspondant aux engagennent déjà pris. ${ }^{14}$ En somme, il s'agit d'une épargne obligatoire; par conséquent, les capitaux mis ainsi en réserve vont obligatoirement s'accumuler.

On estime que, vers 1975 , les pensions servies par ces fonds commenceront à représenter l'équivalent des versements qui entrent dans la caisse. A ce moment-là, le total de l'actif atteindra enviren 100 milliards. Un capital prodigieux, sans aucun doute; cependant, le pouvoir virtuel qu'exerceront ces fonds par l'intermédiaire de leurs investissements boursiers sera encore plus prodigieux. Comme les dirigeants des fonds de retraite doivent s'efforcer de mettre leurs capitaux à l'abri d'une dévaluation, ils les investissent couramment en valeurs mobilières. En ce qui concerne les fonds de retraite qui ne sont pas couverts par une assurance, environ $75 \%$ de leur actif sont investis en bourse et, plus particulièrement, en titres des sociétés géantes dont les prefits se traduisent par de coquets dividendes. Or, $60 \%$ de ces fonds sont pratiquement entre les mains de huit ou neuf banques newyorkaises qui, agissant en mandataires, peuvent ainsi exercer le ároit de vote attaché aux titres. De toute évidence, le jour n'est pas loin où, grâce à ces énormes porte-feuilles, les fonds de retraite privés occuperont une position de contrôle dans les entreprises-clés de l'économie américaine. Cette certitude s'est déjà réalisée dans le cas de Sears \& Roebuck, importante «chaîne » de grands magasins. Les mandataires des employés, à la recherche d'un bon investissement pour les capitaux du fonds de retraite, décidèrent d'acheter des titres de Sears \& Roebuck. Aujourd'hui, e'est pratiquement le fonds de retraite qui contrôle la société.

(14) D'après Carlysle A. Bethel, président de la section fiduciaire de l'Association des Banquiers Américains, on établit actuellement, aux Etats-Unis, jusqu'à 25 programmes de fonds de retraite par jour. En automne dernier, dans un discours prononcé à St-Louis, Mr. Bethel déclara: \& Pour les entreprises n'ayant pas encore de fonds de retraite, le problème n'est pas tant de savoir s'il leur en faut un, que de décider de la forme que ce projet prendra s. St. Louis Globe Democrat, le 7 novembre 1958. 
Tel est done le tableau de la concentration du pouvoir économique dans la société américaine. Tableau peut-être forcé, afin de souligner certains faits mais, de toute manière, on aurait tort de conclure que la concentration entraîne inévitablement toutes sortes de maux. Il y a dix ans, le professeur Summer H. Slichter rappela, à juste titre, que l'Amérique compte plus d'entreprises privées, donc de centres d'initiative privée, que n'importe quel autre pays, que, chaque année, les Américains fondent en moyenne 200,000 affaires nouvelles (sans parler des jeunes fermiers qui créent de nouvelles exploitations), que dans chacune de ces entreprises peut naître une nouvelle idée, une innovation, une invention, qu'au cours des 40 années passées, le nombre des entreprises non agricoles a augmenté plus vite que la population. ${ }^{15}$ Il est certain qu'une multitude de petites firmes réussit fort bien à exister à côté des géants qui dominent l'industrie et que celles-ci soutiennent avantageusement la compétition, grâce à la qualité de leurs produits ou de leurs services. On constate, en plus, un changement significatif en ce qui concerne les grosses et même les moyennes sociétés. Comme l'a remarqué le professeur Peter Drucker, dans la plupart des industries-clés, \& l'entreprise dominante représente aujourd'hui une part nettement plus petite du marché que cela n'était le cas pour l'entreprise dominante il y a vingt-cinq ans $»{ }^{16}$ Ainsi, Ford qui accaparait $60 \%$ du marché en 1925 est revenu aujourd'hui à $20 \%$. La fameuse United States Steel Corporation groupait à l'époque de sa création environ $60 \%$ de la capacité de production d'acier. Aujourd'hui, elle ne dispose plus que de $30 \%$, et à plusieurs reprises, la concurrence des «petits 》 l'a forcée à adopter des innovations techniques qu'elle aurait préféré reculer. ${ }^{17}$

Cependant, le trait caractéristique du système économique américain n'en reste pas moins le «combinat industriel géant, symbole de la révolution que fut l'avènement de la production en série. Révolution qui, le professeur Drucker insiste sur ce point, ${ }^{18}$ n'est pas une question de technologie ni une redistribution de forces physiques, mais « un nouveau principe d'organisation sociale qui impose la séparation de l'ouvrier d'avec le produit et les moyens de production ». Par conséquent, c'est l'organisation plutôt que l'individu qui assure la production dans le système industriel.

(15) The American Economy: Its Problems and Prospects. Knopf, New-York, 1948, pp. 18 et 148 .

(16) The New Society: The Anatomy of the Industrial Order, Harper, New-York, 1950 , p. 344.

(17) Democracy and Progress. MacMillan, New-York, 1950, p. 128.

(18) Ouvrage cité, pages 20,5 et 6. 


\section{Le coût d'un niveau de vie élevé}

Telle est l'organisation de la société industrielle qui, encadrée de devises rappelant les méfaits de la machine dévorante, terrorise tant l'intellectuel européen. C'est, en outre, une situation qui aurait épouvanté Adam Smith.

Pourquoi l'évolution s'est-elle engagée dans cette voie? Comment un peuple libre a-t-il pu admettre une accumulation pareille de puissance économique? Comment ce peuple peut-il tolérer, - et, semble$t$-il, avec une superbe indifférence, - que ce phénomène continue et, même, qu'il s'aggrave, du fait de la politique des investissements boursiers auxquels procèdent ces monstrueux fonds de retraite privés?

Il serait absurde de vouloir expliquer ce gigantisme économique, et le développement de cette oligarchie, par une conspiration d'hommes assoiffés de pouvoir. La réponse est beaucoup plus simple: le peuple américain a librement choisi, - il faudrait même ajouter qu'il a durement travaillé pour obtenir, - un niveau de vie qui exige, dans l'usine moyenne, une mise de fonds en bâtiments, équipement, marchandises, de $\$ 13,281$. par ouvrier. Si nous ne considérons que l'industrie lourde, l'investissement est infiniment plus onéreux: dans une aciérie, par exemple, on arrive facilement à $\$ 25,000$ par ouvrier. Dans l'industrie ohimique, il représente en moyenne la bagatelle de $\$ 38,117$.

L'élévation constante du niveau d'existence exige des efforts tenaces. Au cours des 75 années passées, la population des Etats-Unis a triplé, et l'accroissement de la main-d'oeuvre a été encore plus considérable. Actuellement, le marché du travail doit absorber chaque année un nouveau contingent d'un million de salariés. Cependant, au long de cette période de 75 ans, la production nationale, calculée en dollars de valeur fixe, a presque doublé tous les 20 ans, augmentant en moyenne de $3 \%$ (progressifs) par an. Au total, la production natonale est aujourd'hui 13 fois ce qu'elle fut en 1880. Comme les charges fiscales se sont terriblement accrues, l'augmentation des revenus réellement disponibles est évidemment moins frappante. Pourtant, au cours de ces mêmes 75 années, le revenu moyen, impôts déduits a quand même triplé, passant de $\$ 530$. en 1880 à $\$ 1,710$. en 1956 . Si nous considérons la famille-type, le revenu familial, pendant le même laps de temps, a passé de $\$ 2,200$. à $\$ 5,300,{ }^{19}$

(19) Economic Growth in the United States, ouvrage cité, p. 15. 
Ce résultat a pu être acquis parce que la moyenne de production par heure de travail n'a cessé d'augmenter. Alors que la semaine de travail a été réduite de 63 heures à 42 , l'Amérique produit aujoundhui, par heure de travail, le quintuple de ce qu'elle obtenait en 1880 . L'explication réside en un mot: la fameuse «productivité \$. Seulement, la productivité suppose des machines, et les machines coûtent cher. D'après les calculs du Père Léo Brown, de la Compagnie de Jésus, l'énergie électrique dont disposaient les Etats-Unis en 1902 équivalait à l'énergie d'un «esclave mécanique» par habitant, (hommes, femmes et enfants). Plus loin, il décrit (en 1953): « Probablement, l'ouvrier américain d'aujourd'hui dispose d'une énergie mécanique comparable à la force musculaire de plus de 100 hommes \$. 20

Cest par cette prodigieuse accumulation de capacité de production, et par le financement que celle-ci exige, que l'on peut expliquer le volume de la production américaine. Avec à peine plus de $6 \%$ de la population mondiale, et moins de $7 \%$ des terres, les Etats-Unis produisent et consomment aujourd'hui nettement plus du tiers des marchandises et services disponibles dans le monde entier, et fabriquent presque la moitié des produits industriels du globe. ${ }^{21}$

\section{Dépenses d'outillage et de recherche}

L'outillage qu'exige une telle productivité est certes d'un rendement stupéfiant; malheureusement, il est aussi très, très coûteux. Le marché américain, avec ses 175 millions de clients, s'est accru de 3 millions l'année dernière, mais pour s'y faire une place, il ne faut pas reculer devant la dépense. Une enquête faite en 1951 sur les frais d'installation d'une seule usine moderne, dans les diverses branches de l'activité industrielle, a révélé qu'il fallait faire l'avance de 265 à 665 millions de dollars pour se faire une place dans la sidérurgie, de 500,000 à 2 millions pour s'établir fabricant de chaussures, de 700,000 à 3,5 millions pour être minotier, de 250 à 500 millions pour fonder une usine d'automobiles, et au moins 6 millions de dollars pour fabriquer des stylos. ${ }^{22}$

(20) Social Orientations, Loyola University Press, Chicago, 1954.

(21) USA in New Dimensions, par Thomas R. Carskadon et George Soule. NewYork, MacMillan, 1957, p. 4.

(22) Joz S. Band, Economics of Scale, Concentration and the Condition of Entry in Twenty Manufacturing Industries, American Economic Review, mars 1954, p. 36. 
Quant aux recherches destinées à perfectionner les procédés de fabrication ou mettre au point des produits nouveaux, elles coûtent à lindustrie américaine au moins 8 millions de dollars par an. ${ }^{23}$ Dans la brochure déjà mentionnée de la Du Pont de Nemours, nous apprenons que les recherches de la société sont confiées à 2,200 ingénieurs ou chimistes hautement qualifiés (hommes et femmes), qu'assistent 3,600 spécialistes et techniciens; en 1956, le budget du service était de 77 millions de dollars. «Personne, du moins chez nous, ne s'attend à ce que chaque recherche aboutisse à des résultats commeroialement exploitables, - depuis la création du service, donc depuis de nombreuses années, de tels résultats ont été obtenus tout au plus une fois sur vingt $\mathbf{3}$.

Or, pour l'entreprise résolue à conserver sa part du marché, les recherches, malgré leurs frais énormes, sont une nécessité absolue. Le rapport annuel de la Radio Corporation, par exemple, révèle que, sur un volume total de ventes dépassant le milliard de dollars (en 1955), $80 \%$ des produits ou services ou bien n'existaient pas encore, ou bien n'étaient pas commercialement développés, dix ans plus tôt. Mème observation chez l'Union Carbide and Carbon Corporation: sur un chiffre d'affaires de 1,2 milliards de dollars en 1955, environ un tiers des ventes et presque la moitié des bénéfices nets provenaient de produits qui n'existaient pas dans le commence 15 ans plus tôt. ${ }^{24}$ De même, dans le domaine des méthodes de fabrication, c'est la recherche qui a contribué à l'abaissement du prix de l'aluminium, de \$8. la livre en 1882 , d 96 cents en 1953 (calculé en dollars constants). Pour la cellophane qui valait $\$ 2.65$ la livre en 1924 , on devait arriver à 58 cents en 1955 . (Toutefois, la pression gouvernementale a également joué un rôle dans ce sens, comme nous verrons plus loin.)

L'ingéniosité des chercheurs est nécessaire non seulement pour permettre à l'entreprise de conserver sa part du marché, mais également pour compenser la terrible ponction qu'exerce une masse de consommateurs en augmentation continuelle sur les ressources mondiales. Aujourd'hui, une tonne, ou un fût, ou un mètre-cube de matières premières doit fournir plus de produits, ou acomplir plus de travail, qu'autrefois. Au début du siècle, il fallait, pour produire un kilowatt de courant électrique, environ sept fois plus de charbon qu'aujourd'hui.

(23) D'après C.H. Bartlett, vice-président de Westinghouse Electric, (New York Times, 13 novembre 1958), l'industrie électrique consacre $6 \%$ de son chiffre d'affaires à son programme de recherche $\$$.

(24) Nadler, ouvrage cité, p. 7. 
Voici seulement 38 ans, il fallait deux fois plus d'huile brute pour produire un litre d'essence, et encore était-ce une essence de qualité inferieure. Dans la région des Grands Lacs, les compagnies minières traitent aujourd'hui du minerai de cuivre qu'elles négligeaient 50 ans plus tôt, parce que trop pauvre.

La chasse aux matières premières sans lesquelles l'industrie ne saurait soutenir le niveau de vie élevé des peuples occidentaux implique aujourd'hui des opérations titanesques. Le fer du massif de Mesabi, dans le Minnesota, fut exploité, à l'époque de la découverte du gisement, de la façon la plus simple: on le ramassait, à la pelle. Mais, il y a de cela quelques années, la société Hanna, pour atteindre un filon de minerai, dut assécher un lac canadien. Pour accéder à un gisement vénézuélien, situé loin à l'intérieur des terres, la United Steel construisit une route, draina une rivière et, pour finir, créa sur la côte du Maryland une ville de 75,000 habitants, siège des nouvelles usines de Fairless. Les deux tiers des réserves connues de pétrole se trouvent dans les régions s'étendant au nord et à l'ouest du Golfe Persique. Pour les exploiter, il fallut construire des docks, des routes, des canalisations d'eau potable, importer d'énormes quantités d'équipement, installer sur place des dirigeants et ouvriers qualifiés qui allaient encadrer la main-d'oeuvre locale. A telle enseigne que les capitaux nécessaires dépassaient souvent les possibilités financières d'une seule compagnie, aussi gigantesque qu'elle fût. Aujourd'hui, les anciens intérêts de l'Anglo-Iranienne sont gérés par un consortium où siègent des compagnies anglaises, françaises, hollandaises et américaines. Pour se rendre compte de l'envergure des affaires de pétrole et, par conséquent, des compagnies pétrolières, il suffit peut-être de considérer le coefficient de l'augmentation de la consommation aux Etats-Unis depuis le début du siècle: ce coefficient est de $75 !^{25}$

Plus de produits, à meilleur marché, pour une population en augmentation constante: voici à la fois la cause et le résultat de la création des énormes entreprises d'aujourd'hui, avec l'inévitable corrélatif de la concentration de puissance. Cette puissance économique se maintiendra tant qu'il sera malaisé de construire tout seul une voiture dans le garage familial (si c'était faisable, la voiture reviendrait à peu près a $\$ 25,000$.), et que les gens désireront quand même des voitures. Or, les Américains ont certainement ce désir: plus de 36 millions de familles - à peu près les deux tiers des foyers américains - ont une voiture,

(25) Jennings, ouvrage cité. 
5 millions en possèdent deux ou davantage, pour parcourir en moyenne 3,170 milles par an. Pour qu'une telle motorisation fût possible, il fallait l'entreprise géante: l'actif de la General Motors atteint presque 7 milliards de dollars. Son chiffre d'affaires net en 1955, - $\$ 12,443$, 277,420. - dépassait le budget total de la France (3,645 milliards de francs) et représentait environ $93 \%$ du budget britannique $(£ 4,738,000$,000.). Du moment que l'on veut pouvoir disposer librement de quelque 50 millions d'appareils téléphoniques, il faut bien accepter l'existence d'un monopole à peine inavoué tel que celui de l'American Telephone and Telegraph Company, avec un actif de $\$ 17,765,876,672$ qui fait de cette société un organisme plus riche que biens des Etats souverains membres des Nations Unies.

Ceci dit, il ne faudrait pas considérer les paragraphes précédents comme un crédo de la nécessité des entreprises géantes, seules capables d'atteindre et de maintenir les rendements indispensables. Sur ce point, les économistes sont loin d'être d'accond. ${ }^{26}$ En outre, la puissamce des oligarchies constitue toujours une menace politique: il suffit d'imaginer ce qui se produirait le jour où le bien de la nation américaine et celui de la General Motors se confondraient dans l'esprit d'un industriel nommé secrétaire d'Etat. Le rapport majoritaire du comité anti-trust et anti-monople du Sénat, publié le 6 novembre dernier, invitait le Département de la Justice à examiner de près la structure et l'activité justement de la firme à laquelle Mr. Charles Wilson (secrétaire d'Etat à la Guerre) appartenait jusqu'à sa nomination. Nombreux sont ceux qui estiment qu'une « décartellisation» de la General Motors, c'est-d-dire le retour à l'indépendance des firmes qui la composent, ferait baisser le prix des voitures et, en outre, aboutirait à la création de modèles mieux adaptés a $\mathbf{x}$ désirs de la clientèle. Si la société voulait se donner la peine de répondre, elle expliquerait sans doute que, pour citer le professeur Drucker, elle constitue une « fédération 》, une association d'unités alliées mais autonomes qui se font mutuellement concurrence, pour le plus grand profit des clients. ${ }^{27}$

\section{Le gigantisme est-il inévitable?}

Il existe un homme qui discute régulièrement avec la General Motors et qui, pourtant, ne semble nullement préoccupé par les dimensions colossales de son redoutable interlocuteur. Cet homme, Mr.

(26) Le rapport entre les dimensions et le rendement d'une entreprise est une question controversée (Slichter, ouvrage cité, p. 16).

(27) Ouvrage cité, pp. 269-276. 
Walter Reuther, grand maittre du syndicat des travailleurs de lautomobile, déclarait récemment à un journaliste britannique:

< Pour commencer, il faut admettre, je pense, qu'étant donné la technologie moderne, les entreprises géantes sont un phénomène inévitable. On peut imaginer de petites entreprises lorsque la situation technique reste relativement simple; mais à Y'ère de T'automation, de l'électronique et, (dans le lointain) de l'utilisation pacifique de latome, lâ petite entreprise devient une impossibilité.

Je m'explique: à mesure que la technologie devient plus complexe, qu'il faut des investissements toujours aocrus pour fournir du travail à un seul ouvrier, l'industrie marchera dans le sens de la concentration, - que cela nous plaise ou non.

La vieille conception libérale d'après laquelle l'entreprise énorme est la source de tous les maux, et le petit atelier le siège naturel de toutes les vertus, - cette conception n'est qu'un ramassis de fadaises. On peut avoir affaire à de petits tyrans comme à de grands tyrans. Les grands syndicats sont un sous-produit des mêmes phénomènes technologiques qui ont engendré la grande industrie. On ne voit pas très bien un petit syndicat discuter avec la General Motors; pour répondre à une telle puissance, il faut des syndicats énormes et puissants.

Il ne s'agit pas de savoir si nous voulons nous évader d'un univers démesuré, avec ses immenses entreprises, ses immenses syndicats, ses immenses gouvernements. La question à résoudre, la voici: Comment vivre avec ces géants, et les amener à tenir compte des besoins élémentaires de l'homme?

Vous me demandez comment nous allons éviter que l'individu ne soit égaré dans la bousculade, dans la jungle des Grands? Ma foi, c'est une question qu'il faudra prendre le temps de penser jusqu'au bout ».

Il est certain que cette dernière question exigera, en effet, qu'on «prenne le temps de la penser jusqu'au bout 》. Ce qui s’applique également à la possibilité d'une co-existence des petites entreprises avec les géants, et plus particulièrement aux chances qu'aurait une nouvelle affaire de naître et de prospérer. D’après Drucker, «ce qui importe est non l'envergure comparée des entreprises, mais le renouvellement constant des cellules économiques. Il s'agit donc du métabolisme basal de la société industrielle ». ${ }^{28}$

(28) Drucker, ouvrage cité, p. 344. 
En somme, pour que notre société libre soit assurée d'une bonne santé, il faudrait élaborer une politique positive et énergique d'encouragement aux nouveaux venus. De même, dans l'agriculture, il faudra reconnaître l'importance de la ferme familiale. ${ }^{20}$

Cependant, bien que nous préférions en théorie un régime où la propriété privée serait très largement répartie, nous sommes bien forcés d'admettre que le gigantisme est la marque de la société industrielle d'aujourd'hui. De même, le «nouveau principe de l'organisation sociale» répond au désir d'un niveau de vie plus élevé. (Je sais bien qu'en insistant sur ce dernier point, je risque d'être aocusé de «matérialisme», surtout par ceux qui trouvent une noblesse mal définie dans la cueillette du coton à la main, et qui crient à la «dépersonnalisation 》 lorsqu'on emploie une moissonneuse. Quant à savoir comment la population actuelle du globe qui a doublé en moins d'un siècle pourrait être nourrie et vêtue sans le secours de l'industrie moderne, c'est une question qui dépasse l'entendement). ${ }^{30}$

Ceci admis, la concentration de la puissance économique reste un phénomène troublant pour l'esprit habitué aux préoccupations sociales du catholicisme et à la tradition politique américaine. Aujourd'hui encore, il peut être avantageux, pour un politicien américain, d'attaquer * les géants de l'industrie». Les Etats-Unis ont, déjà, un long passé d'enquêtes parlementaires sur l'activité des trusts ou ententes professionnelles. A la plus belle époque du règne de Roosevelt, le Congrès institua un Comité économique temporaire, chargé d'étudier la concentration industrielle. Après trois années d'effort, concrétisés par 17,000 pages imprimées, le comité arriva à la conclusion que l'on observait effectivement une concentration très poussée dans l'industrie américaine. Il préférait cependant s'abstenir de toute suggestion parce que:

«Les membres du comité ne sont pas assez téméraires pour se croire capables d'établir un programme susceptible de résoudre des problèmes immenses qui se posent dans le monde entier. En revanche, ils sont convaincus que les éléments réunis par eux permettront au

(29) Voir Social Order, novembre 1958.

(30) Au recensement d'octobre 1958, les Etats-Unis comptaient 175 millions d'habitants, 20 millions de plus qu'au moment de l'élection d'Eisenhower, un accroissement presque égal à la moitié de la population française ou anglaise. D'après les travaux du Centre Démographique de Chicago, l'Amérique comptera en 1975 entre 216 et 244 millions d'habitants. Ouvrage cité, pp. 13-16 et 197. 
peuple américain de comprendre ce qu'il faudra faire pour préserver les libertés humaines ».

Un acte de foi vraiment touchant: le comité compte sur la sagesse innée du peuple américain. Ou encore, si l'on veut se montrer sarcastique, une belle démonstration de l'art de se débarrasser d'une responsabilité.

\section{Mesures pour établir l'équilibre}

L'auteur du présent article se propose de démontrer que, malgré la concentration de la puissance économique résultant de l'avènement de la production en série, les libertés humaines ont été effectivement préservées. Et cela grâce à un ensemble de restrictions légales, à l'apparition d'institutions de compensation et, aussi, à la force de nos traditions politiques et culturelles. L'auteur estime également qu'en tant que bouclier des libertés, garantie de la sécurité matérielle et stimulant de l'initiative, ces facteurs sont infiniment précieux. A son sens, ils peuvent fort bien remplacer le régime préconisé par Jefferson et les « distributionnistes » modernes, régime basé sur la large répartition de la propriété privée, en ce qui concerne la terre comme pour l'outillage.

On pourrait me répondre que nous assistons au passage du régime de la propriété à celui de la puissance. Tout comme la répartition de la propriété était une condition essentielle du maintien des libertés fondamentales, de la sécurité matérielle et des possibilité de développement de lindividu, la répartition de la puissance est nécessaire au maintien des mêmes principes. Dans l'Amérique contemporaine, cette répartition est accomplie, du moins dans une mesure à peu près suffisante.

Malgré les rumeurs au sujet d'un danger fasciste, aux plus beaux jours de feu le sénateur MacCarthy, l'avenir de nos libertés politiques ne semble guère menacé, de quel côté qu'on se tourne. "Mettons les braillards à la porte», telle est la solution traditionnelle aux EtatsUnis dès qu'un parti politique installé au pouvoir devient trop insolent. Les élections et la Cour Suprême chargée d'interpréter notre Constitution se sont toujours montrées capables de protéger les droits de l'individu, et de rendre superflue toute tentative révolutionnaire du prolétariat dépossédé. Notons en passant que le groupe de citoyens 
dont les droits civiques sont violés de la façon la plus flagrante, c'està-dire les Noirs, ne trouve guère de protection dans la propriété. Au contraire: un indice extérieur de réussite matérielle, comme l'aoquisition d'une maison dans un quartier plus agréable, accroit souvent la résolution du groupe dominant d'opprimer davantage la minorite, de « veiller à ce que ces gens restent à leur place ». La défense des droits civiques des Noirs a été réalisée grâce aux décisions de la Cour Suprême, au législateur et à l'éducation du public.

Quelles sont donc ces forces d'équilibre, ces contrepoids si l'on préfère, ${ }^{31}$ qui ont créé un milieu où la puissance économique est contenue dans des limites acceptables, où la croissance économique et l'élevation du niveau de vie se déroulent sous le contrôle de l'Etat, mais sans que celui-ci intervienne par quelque plan autoritaire? $\mathrm{Ce}$ sont les restrictions légales, les préférences des consommateurs, les pressions de certains groupements et, surtout, les effets lents mais tenaces de la tradition politique américaine qui ont créé cet équilibre et permettent d'en espérer la pérennité.

Examinons donc le fonctionnement de ces contrepoids, observonsles dans leur effort de création et de soutien d'un milieu où la puissance est répartie.

De toutes les forces qui s'opposent aux éventuels excès des grandes concentrations de puissance économique, la plus visible, la plus manifeste est sans doute l'action du gouvernement fédéral. Celui-ci intervient à la fois par sa politique fiscale et par son oeuvre de surveillance.

\section{L'intervention gouvernementale}

Les impôts et taxes ont été utilisés non seulement pour couvrir les dépenses de l'Etat, mais aussi en vue du but social qu'est la répartition de la puissance économique.

Une taxe fédérale de $52 \%$ frappe les profits des sociétés supérieurs à $\$ 25,000$. Quelque $30 \%$ du revenu national sont absorbés par le fisc. Citons ensuite la législation anti-trust, du Sherman Act de 1890, destiné à prévenir toute emprise à caractère de monopole sur un marché

(31) Nous avons délibérément évité le terme de \& forces adverses s. Nous lui donnons, en effet, dans cet exposé une signification plus vaste (et d'ailleurs différente) de celle que le professeur Galbraith attribue à cette expression dans son Capitalisme Américain. 
donné, à la loi anti-cartel de 1950. La façon d'utiliser ces textes législatiffs a toujours donné lieu à d'âpres discussions: à en croire certaines critiques, ces lois pénalisent les hauts rendements, reprochent à l'envergure d'avoir de l'envergure et condamne avec une sévérité excessive des entraves purement théoriques au commerce. Il n'en reste pas moins que cette législation, par sa seule existence, constitue une menace constante à l'adresse de certains hommes d'affaires tentés d'adopter une conduite anti-sociale. C'est ainsi qu'elle a été utilisée par le Département de la Justice pour contraindre, par exemple, l'industrie cinématographique à liquider des théâtres, la Compagnie Pullman à cesser de s'intéresser aux fabrications de wagons-lits, et la Telephone and Telegraph Company à réduire le prix des communications interurbaines. Au printemps dernier, en l'espace d'une seule journée, l'administration entama des actions anti-trust contre cinq grosses entreprises, dont l'une était accusée d'avoir établi un monopole international dans un domaine singulier: la fabrication des manches de club de golf. ${ }^{32}$

Le gouvernement dispose, pour surveiller les banques et organismes finanoiers, de la Loi pour la Garantie de la Monnaie (Federal Reserve Legislation), pour les transports ferroviaires et routiers de la Loi sur le Commerce entre Etats, (renforcée par des textes ultérieurs), pour l'énergie et la lumière électrique, de la Loi Fédérale sur l'Energie, pour la radio et la télévision de la Loi sur les Communications, pour le pétrole et l'essence du Règlement Pétrolier Inter-Etats et de la loi Conally, pour les transports fluviaux et maritimes des lois de la Commission Maritime, pour les opérations boursières de la loi sur les Valeurs Mobilières, pour la production de la viande de la loi sur la Boucherie et les Abattoirs, pour l'aviation de la Loi de l'Aéronautique civile, pour les raffineries de sucre d'une loi spéciale de 1948 qui autorise le secrétaire d'Etat à l'Agriculture à fixer les quantités du sucre à importer.

Après la guerre, le gouvernement passa à l'attaque contre le monopole de l'Aluminum Company of America. Grâce à l'emploi judicieux des menaces légales et à une politique d'aide financière, on put installer équitablement, dans cet important domaine, deux concurrents d'envergure, la Reynolds Metal Company et la Kaiser Aluminum Company. De même, le groupe Studebaker-Packard fut pratiquement sauvé de la disparition par un prêt fédéral accordé à Curtiss-Wright qui put ainsi acquérir la vieille firme qui battait sérieusement de l'aile.

(32) Business Week, 5 juillet 1958. 
En théorie, la principale entrave à la puissance économique privée est la concurrence. Quant à savoir dans quelle mesure la concurrence existe encore dans l'économie américaine, c'est un sujet de discorde pour les spécialistes. On parle beaucoup de «prix embrigadés », c'està-dire de prix qui seraient fixés par les entreprises dominant le marché, sans qu'il y ait vraiment collusion, mais aussi sans que l'on tienne compte de l'offre et de la demande. ${ }^{33}$ On souligne que toutes les grandes marques de cigarettes sont vendues au même prix; dans la compétition pour la conquête d'une plus grande part du marché, les fabricants recourent surtout à une publicité agressive et à des emballages plus attrayants. Récemment, la véritable oligarchie qu'est l'industrie des moteurs fit l'objet d'une enquête du Congrès pour déterminer si et dans quelle mesure les fabricants s'entendaient pour la fixation des prix.

Il est évident que la compétition conforme au pur idéal d'Adam Smith et de Jean-Baptiste Say n'existe pas dans des marchés que dominent un très petit nombre d'entreprises géantes. Aussi souhaitable qu'elle soit, et malgré tous les encouragements possibles et imaginables, elle n'a jamais eu d'existence réelle que dans les manuels. Et pourtant, le consommateur reste roi; ne serait-ce que parce qu'il garde la possibilité de ne pas acheter (à condition, bien entendu, qu'il puisse résister à la préoccupation lancinante de «ne pas se laisser épater par le voisin»). Ainsi, le consommateur peut décider, par exemple, d'acheter une Ford plutôt qu'une Chevrolet, mais il décidera également, en toute liberté, de vendre ou de garder sa vieille voiture. Les offres des marchands de voitures d'occasion interviendront évidemment dans son choix: favorables, elles l'inciteront à changer de voiture, peu favorables, à attendre encore une année.

Dans lindustrie automobile, la concurrence garde au moins cette réalité: les diverses sociétés cachent soigneusement leurs modèles en préparation, elles les entourent du plus grand secret, afin de conser-

(33) Le problème a été longuement examiné dans "Pricing in Big Business ?, publié en 1958 par The Brookings Institution, Washington. Dans cet ouvrage, les auteurs, A.D. Kaplan, Joel B. Dirlam et Robert F. Lanzolloti, étudient 20 grandes entreprises américaines. Ils constatent que les prix sont \& embrigadés » dans ce sens qu'ils sont déterminés d'avance, selon le prix de revient probable et les perspectives du marché. Les grandes sociétés, si elles évitent de se concurrencer directement dans le domaine des prix, emploient d'autres moyens pour se faire une guerre acharnée. Les fabricants de voiture rivalisent par leurs modèles, les usines d'équipements agricoles par l'ingéniosité et la façon de servir le client, les géants de l'industrie chimique par l'assistance technique offerte gracieusement aux acheteurs, etc. Les auteurs admettent «leur incapacité de classer les prix dans une même catégorie \$. 
ver l'avantage de telle ou telle particularité qui pourrait attirer les acheteurs. A Détroit, la rivalité des Trois Grands de l'automobile est telle que leurs dirigeants s'inscrivent délibérément dans des clubs différents. Ils ont d'ailleurs, du moins jusqu'aux dernières négociations pour un nouveau contrat collectif, refusé de s'entendre pour opposer un front uni au syndicat de Walter Reuther. Il est certain que, dans ce farouche désir d'indépendance, la crainte d'un gouvernement toujours à l'affût d'une collusion illégale, et une grande sensibilité à l'opinion publique, jouent un rôle bien plus grand que la rivalité commerciale proprement dite. Et pourtant, la concurrence existe, même dans l'oligarchie de l'automobile. Le succès du modèle "Rambler», de American Motors, et l'accroissement des importations d'Europe ont forcé chacun des Trois Grands à lancer un programme-éclair afin de produire une petite voiture pour laquelle il y a aujourd'hui un marché tout prêt.

De même, il existe certainement une «concurrence par la nouveauté » qui, d'une part, fait fonction de frein imposé à la puissance économique privée et, de l'autre, peut devenir un stimulant pour la recherche. Dans de nombreux cas, les matières plastiques, les produits laineux, le verre, l'aluminium ou d'autres métaux non ferreux ont remplacé l'acier. Un changement dans les goûts de la clientèle peut aboutir à l'élimination d'une firme qui manque d'esprit d'initiative. Ainsi, la télévision a failli tuer le cinéma; au dernier moment, Hollywood découvrit qu'on pouvait vendre des archives de vieux films à ce terrible rival, qu'on pouvait même utiliser les studios pour tourner des programmes de télévision. De même, un changement dans les habitudes de la clientèle peut transformer une industrie. L'automobile a permis aux employés et fonctionnaires de vivre dans les nouveaux faubourgs-champignons qui poussent à la lisière des grandes villes elle les a du même coup libérés de la servitude des transports en commun, si bien qu'aujourd'hui, les compagnies d'autobus et de tramways perdent de l'argent. ${ }^{34}$ Depuis que les lignes d'aviation ont rattrapé les chemins de fer en ce qui concerne le nombre des voyageurs-kilomètre (25 milliards par an), il a fallu réduire considérablement la circulation ferroviaire afin de diminuer les pertes, et les chemins de fer ont même demandé des exemptions fiscales.

(34) Les réseaux interurbains reliant des villes de plus de 500,000 habitants ont enregistré, entre 1946 et 1957 , une diminution de $48.9 \%$ du nombre des voyageurs. 


\section{La puissance des syndicats}

Le mouvement syndical constitue, lui aussi, un contre-poids de taille aux concentrations de la puissance industrielle. Il représente d'ailleurs lui-même une énorme puissance économique qui, d'après le professeur Slichter, symbolise fort bien notre économie. ${ }^{35}$ Avec leur 18 millions de membres, les syndicats américains détiennent aujourd'hui une puissance telle que l'opinion publique approuve le plus souvent les enquêtes sénatoriales sur leurs abus de pouvoir. Les syndicats ont obtenu de meilleures conditions de travail et des salaires plus élevés, à telle enseigne que de nombreux économistes voient dans le prix de la main-d'oeuvre la cause première d'une inflation aussi inofficielle que constante. Malgré les pertes de salaire résultant de la dernière « récession», la plus grave depuis 1949, le revenu national augmentait de $2.5 \% .{ }^{3 \mathrm{~B}}$ Un fait qui cesse d'étonner quand on sait que, pendant cette période, les revisions des contrats collectifs améliorèrent les conditions d'existence de 850,000 ouvriers. ${ }^{37}$ Le ler Juillet 1958, en pleine crise, les ouvriers de la sidérurgie obtinrent automatiquement une augmentation de salaire et de primes variant de $16.9-19.1$ cents de l'heure. Même la General Motors, avec un actif de près de 7 milliards de dollars, a le plus grand respect pour la puissance du syndicat des travailleurs de l'automobile.

Il n'est sans doute pas inutile de rappeler ici que le mouvement syndicaliste américain comptait essentiellement sur la négociation avec le patronat pour obtenir le droit de participer au façonnage de l'économie nationale. Ce ne fut que tout récemment que les syndicats s'aventurèrent dans l'arène politique. ${ }^{38}$ George Meany, président de la centrale syndicaliste AFL - CIO, a d'ailleurs déclaré que «cette organisation a surtout un caractère économique, et elle s'efforce d'agir dans le domaine économique ».

«Les buts que nous poursuivons en tant que syndicalistes, poursuivit-il, sont plutôt modestes, car nous ne cherchons nullement à réformer la société américaine selon telle ou telle doctrine. Nous désirons jouir d'un niveau de vie en constante amélioration. Ce désir, Sam Gompers l'exprima, un jour, par une formule particulièrement

(35) Cette économie est fréquemment définie comme celle de la libre entreprise. Certainement un nom mal approprié.

(36) Business Week, le 8 mars 1958, p. 126.

(37) Monthly Labor Review, août 1958, p. 899.

(38) Voir Francis X. Quinn, s.j., Labor at the Polls, Social Order, novembre 1958, pp. 412-419. 
concise. Comme on lui demandait ce que voulaient les syndicats, il répondit simplement: «Davantage »; même si nous entendons, sous le terme e élévation du niveau de vie», non seulement plus d'argent, mais aussi plus de loisirs et même la possibilité d'accéder à un meilleur niveau intellectuel, le principe reste. Ce que nous réclamons? \& Davantage s. ${ }^{\text {so }}$

Ce qui montre bien que le syndicalisme américain ne s'embarrase guère de considérations idéologiques.

\section{Les syndicats acceptent le système}

Invité à prendre la parole au Congrès de l'Industrie Américaine, en décembre 1955, sur le sujet «Ce que les Syndicats attendent des Dirigeants », Mr. Meany déclara:

- Dans l'ultime analyse, nous ne constatons pas une grande différence entre la cause que nous soutenons, et celle que soutient l'Association Nationale des Chefs d'Entreprise. Moi, je soutiens le système basé sur la recherche du profit, pour la bonne raison que j'y crois. A mon sens, c'est le meilleur des stimulants. Je crois en le système de la libre entreprise, je lui fais confiance sans aucune réserve. Je crois en la rétribution du capital investi, je crois en le droit de la direction de diriger».

(La même année, mais un peu plus tôt, dans un article publié par la revue Fortune, Mr. Meany affirmait: « Je le dis carrément: la négociation d'un contrat collectif n'est pas un moyen de s'introduire dans la direction de l'affaire. Nous ne souhaitons pas ce qu'on appelle la co-gestion, - la représentation des syndicats dans les conseils d'administration ou dans la direction technique de l'entreprise »).

Après avoir ainsi rassuré les hommes d'affaires, en proclamant son adhésion totale au système économique américain, le président de la nouvelle centrale syndicale (née de la récente fusion de l'AFL et de la CIO) posa à son public d'industriels la question suivante:

- Croyant en tout cela, en tant que représentant des syndicats libres, quels sont les sujets de discorde qui subsistent entre nous?

(39) What Labor Means by 'More', \& Fortune \$, mars 1955, p. 92. 
Uniquement le désacoord portant sur les parts respectives du travailleur et de la direction dans les biens produits par l'entreprise. En ce qui me concerne, je suis certain qu'on trouve assez d'intelligence ohez l'ouvrier américain comme chez le dirigeant américain pour résoudre cette question d'une façon équitable, bien américaíne ${ }^{40}$

Attitude qui paraît bien éloignée des slogans virulents et mème révolutionnaires des chefs syndicalistes d'Europe. On la condamnera peut-être, non seulement pour son manque de solidarité ouvrière, mais aussi parce qu'elle traduit l'opulence du représentant grassement rétribué. Personnellement, je croirais plutôt que l'attitude de Mr. Meany illustre l'absence totale d'une conscience de classe aux Etats-Unis. ${ }^{41}$ Or, affirmer que l'Amérique constitue une société sans classes, c'est amplifier le fait d'une communauté de conceptions, par exemple au sujet du rôle que doit jouer le gouvernement, de la forme idéale de la société, du respect des droits de l'individu (nous passerons pour l'instant sur la honte nationale de la ségrégation, problème qu'on finira obligatoirement par résoudre, sous la pression permanente de notre Constitution qui proclame l'égalité politique de tous les citoyens). Cette communauté d'idées et d'attitudes résulte d'une expérience historique commune, du fait d'appartenir à un peuple qui n'a pratiquement pas de passé. ${ }^{42}$ Le Président Roosevelt souligna, un jour, ce fait dans un discours devant l'Assemblée des Filles de la Révolution Américaine, groupement strictement conservateur: «Chers Compagnons d'Immigration, commença-t-il...

Un récent «Gallup » portant sur la catégorie sociale à laquelle les personnes interrogées pensaient appartenir révéla que $90 \%$ des gens ayant accepté de répondre se rangeaient dans les «classes moyennes 》. Il est probablement vrai, comme on l'a déjà dit, que l'Amérique devient,

(40) Brochure publiée par le National Association of Manufacturers, New York 17.

(41) Le professeur Clair Wilcox affirme: \& La lutte des classes en Amérique n'oppose pas le prolétariat à la bourgeoisie. C'est une lutte entre groupements fonctionnels possédant chacun une puissance fortement concentrée - une lutte pour le contrôle de la production industrielle ». Wilcox énumère ces groupements: les Grands Syndicats, la Grande Agriculture, la Grande Entreprise. Sa thèse illustre les efforts et l'emplacement des principaux contrepoids qui freinent la puissance économique et, je le prétends, tiennent la place que la pensée sociale catholique assignait autrefois à la propriété privée.

(42) La ville où eut lieu le Congrès - Berlin, capitale écartelée - rappelle à l'auteur l'oeuvre où Goethe, sous le titre \&Aux Etats-Unis », s'exclame: *Amérique, tu es bien mieux lotie que notre vieux continent: tu n'as ni ehâteaux ruinés, ni souvenirs stériles, ni querelles inutiles \$. 
politiquement, une nation de conservateurs, dans ce sens qu'un nombre toujours croissant d'individus accède à ce niveau économique où l'on a quelque chose à conserver. D'ailleurs, il est tout aussi évident que les personnes appartenant à une même classe s'attendent à être traités en égaux.

Si bien que l'arbitrage devient «le procédé-type pour résoudre les conflits relatifs à linterprétation des contrats collectifs, lorsque les parties en présence ne parviennent pas à se mettre d'accord \$. ${ }^{43}$ Les avantages de la méthode sont évidents. D'abord, les conflits entre syndicat et direction se trouvent soumis au jugement d'un tiers impartial. Mais, en plus, certains syndicats ont établi des listes de personnes respectables et respectées auxquelles n'importe quel adhérent peut s'adresser lorsqu'il est en désaccord avec les chefs du syndicat. Le Père Leo C. Brown, de la Compagnie de Jésus, fait partie du jury d'appel des travailleurs de l'automobile.

Cette interdépendance des grandes entreprises et des grands syndicats agit comme un frein qui s'oppose aux excès éventuels de la puissance économique. Situation extraordinaire et, dans un certain sens, unique, constate une enquête de la National Planning Association:

«Les syndicats d'Europe Oocidentale ont délibérément tenté d'amener des changements dans la société. Or, à l'exception des pays scandinaves, remarquables par leur stabilité, et dont nous ne parlerons pas ici, ces efforts n'ont abouti quà des résultats très restreints. Les syndicats américains, fidèles aux principes de Samuel Gompers qui leur assigne une action purement économique, n'ont pas cherché à transformer la société; en revanche, ils ont directement contribué à lacomplissement de certaines transformations fondamentales, conséquences de leur action économique ». ${ }^{4}$

A quoi l'observateur européen répondra qu'en réduisant les syndicats au rôle d'une organisation purement économique, on leur dénie des responsabilités plus vastes dans la recherche du bien commun. (Cet Européen sera peut-être tenté d'oublier le rôle des syndicats américains qui ont soutenu le Plan Marshall, l'aide aux pays sous-développés, l'accueil plus libéral des immigrants, ainsi que des projets communautaires d'intérêt local.) Que fait-on, demandera notre Européen, de

(43) Voir Leo C. Brown, s.j., Labor Arbitration, Social Order, février 1957, p. 62.

(44) Trade Unions and Democracy, une étude comparative des syndicats américains, français, italiens et ouest-allemands, Washington, 1957. 
la participation syndicale dans l'étude d'ensemble de l'économie nationale, de sa situation présente et de ses perspectives d'avenir?

On aura beau lui citer des groupements tels que le Comité pour le Développement Economique, ou la National Planning Association, il répliquera qu'il s'agit là d'organismes privés, sans aucune responsabilité publique. De même, il repoussera le Comité des Conseillers Economiques du Président des Etats-Unis, qu’il considérera comme l'instrument de l'administration politique. L'idéal de notre observateur européen serait quelque chose dans le genre du Conseil Economique français qui groupe des représentants du gouvernement, des syndicats, des patrons, ainsi que des experts indépendants appartenant à l'Université, et qui étudie les grands problèmes de la politique économique. Même la suggestion du Professeur Burns qui propose de fondre les 14 organismes de l'Advisory Board of Economic Growth en un seul Conseil Economique lui paraîtra insuffisante.

En revanche, il sera fort intéressé par une proposition d'Arthur J. Goldberg, conseiller de la grande centrale syndicale AFL-CIO. M. Goldberg, dans un discours prononcé à l'université de Wisconsin, envisageait un organisme groupant syndicats et dirigeants patronaux, et qui se réunirait, sous lautorité bienveillante mais non la surveillance du gouvernement, pendant deux ou même trois semaines, une ou deux fois par an, afin d'étudier les grands problèmes communs: niveau des prix et profits, automation, si les salaires dépassent le volume de la production ou non, retraites ouvrières, etc.

Proposition qui semble constituer un premier pas bien timide vers l'arrangement social suggéré par le regretté pape Pie XII au Congrès de l'Union Internationale des Patrons Catholiques qui eut lieu au Vatican, en mai 1949:

« Notre inoubliable prédécesseur Pie XI préconisait déjà, dans Son encyclique Quadragesimo Anno, une «organisation fonctionnelle» dans les diverses branches de lindustrie. Rien ne lui paraissait plus apte à assurer le contrôle du libéralisme économique que la création d'un statut légal, basé justement sur la responsabilité collective de tous ceux qui concourent à la production. Pourquoi serait-il interdit d'assigner aux travailleurs leur juste part de responsabilité dans l'établissement et le développement de l'économie nationale? (Catholic Mind, juillet 1949, p. 446.) 
La proposition de M. Goldberg reste certainement assez éloignée du but esquissé par le professeur Frank Tannenbaum, de l'Université de Columbia, auteur de l'ouvrage Philosophie du Syndicalisme (Knopf, New York, 1951). Tannenbaum estime que «l'économie actuelle ne saurait reposer éternellement sur un système de propriété fluide, impersonnelle, et de travail fluide, impersonnel». Il prévoit que «les syndicats assumeront progressivement le rôle d'une corporation moderne, en achetant des participations dans leurs corporations respectives, et que la propriété cessera d'être fluide et impersonnelle». En conclusion, « la corporation et le syndicat finiront par se fusionner, par se confondre dans une propriété commune, si bien que leur co-habitation sera libérée de toute hostilité ».

\section{La force de I'opinion publique}

De même, l'opinion publique parvient à modérer l'influence de la concentration économique. Il y a, tout d'abord, la crainte très réelle d'indisposer la masse des clients éventuels par une attitude ou action que ceux-ci risquent de condamner. Ainsi, au printemps dernier, l'industrie automobile semblait occuper une position extrêmement forte vis-à-vis de son puissant syndicat. Avec 800,000 voitures invendues, avec un chốmage de plus en plus alarmant dans la région de Détroit, les sociétés paraissaient en mesure d'imposer leur volonté à Walter Reuther, retournant contre lui sa tactique habituelle de «Pas de contrat, pas de travaill» Or, bien que le contrat collectif arrivât à expiration, les sociétés continuèrent à appliquer l'augmentation périodique des salaires, en bonne partie par crainte de l'opinion publique. (Un autre facteur affaiblissait certainement l'apparente position de force de la General Motors, fournissant un nouvel exemple de l'action des contrepoids: la société avait de bonnes raisons de redouter que le gouvernement n'entamât contre elle une action anti-trust, au cas où elle aurait provoqué une crise en pleine période de récession.)

Dans plus de 4,000 grandes entreprises, les chefs des public relations doivent non seulement expliquer aux clients la politique suivie par leur firme, mais aussi expliquer à leurs patrons ce que les clients attendent d'eux. Comme disait Paul Garrett, de la General Motors: * Nos fonctions consistent en ceci: découvrir ce que les gens aiment, le faire davantage, découvrir ce qu'ils n'aiment pas, et en faire moins 》. Le public considère les entreprises comme une partie de la communauté; par conséquent, les entreprises ont dû apprendre à accepter leurs 
responsabilités sociales. John S. Coleman était président de la Chambre de Commence des Etats-Unis quand il déclara: «De nos jours, le patronat voit son travail avec les yeux de la communauté, et envisage le bien commun, en s'efforçant de regarder haut et loin ». Citons également l'attitude de J.D. Zellerbach, président de la deuxième usine de papier d'Amérique et l'un des leaders du Comité pour le Développement Economique. Aujourd'hui, Mr. Zellerbach est ambassadeur d'Amérique d Rome. Ce fut lui qui déclara, voici quelques années:

- Si la majorité des Américains sont pour l'entreprise privée, ils ne la considèrent pas comme un droit naturel, mais simplement comme le meilleur moyen de travailler utilement dans une société libre. A leur sens, les patrons doivent se comporter en fidèles et loyaux gérants qui dirigent l'économie pour le plus grand bien de la communauté s.

Langage qui peut sembler prétentieux et hypocrite lorsqu'on ignore les changements intervenus dans la fonction de dirigeant. David Lilienthal, observateur perspicace de l'évolution sociale et, en mème temps, modèle parfait du haut fonctionnaire, s'est exprimé ainsi: \& De nos jours, le travail du directeur d'une grosse entreprise doit ressembler davantage à celui d'un fonctionnaire qu'aux occupations du patron d'autrefois ». Le fait est que l'entreprise devient presque une forme de gouvernement privé, avec son «peuple» d'actionnaires, de fournisseurs, clients et salariés. Clarence Francis, président du conseil d'administration de la General Foods, l'une des plus grosses sociétés d'Amérique, remarque à ce propos:

- Aujourd'hui, la majeure partie du patronat tient compte des désirs de ses salariés, de ses clients, et même des directives gouvernementales. Il s'agit donc d'équilibrer ces forces, et de veiller à ce que chaque catégorie reçoive une juste part des profits ».

Mr. Francis aurait pu ajouter à cette énumération les désirs de la communauté locale où l'entreprise est installée. L'opinion publique estime que le patron doit s'intéresser aux affaires publiques, notamment aux activités civiques et sociales. Si bien que le dirigeant «moyen», à la fois pour le bien de l'entreprise et par conviction personnelle, consacre aujourd'hui jusqu'au tiers de son temps aux oeuvres communautaires, et en exige autant de ses subordonnés. On lui demandera de donner non seulement son argent mais aussi son énergie et ses compétences aux campagnes destinées à collecter des fonds pour la 
Croix Rouge, l'orchestre symphonique de la ville (il en existe 170, aux Etats-Unis), l'hôpital municipal, les églises, les musées (dont on compte quelque 2,500 en Amérique), la lutte contre le cancer, les affections cardiaques, la tuberculose, la poliomyélite, la paralysie cérébrale et, à ne pas oublier, pour les collèges et universités, dont la majeure partie sont des institutions privées, aux Etats-Unis.

Pendant ces deux dernières années, les grandes sociétés ont donné aux oeuvres philantropiques un peu plus d'un milliard de dollars. Chaque année, elles contribuent pour plus de 100 millions au budget de l'instruction secondaire et universitaire des institutions privées. Le gouvernement, de son côté, encourage ces libéralités en autorisant la déduction, du total imposable, de $5 \%$ des gros bénéfices, du moment que ces sommes sont versées à des oeuvres d'intérêt public.

En 1956, la Fondation Ford, dont le capital est investi en actions de la société, offrait 500 millions de dollars pour l'amélioration de l'enseignement de la médecine, l'entretien des hôpitaux et, plus généralement, le financement des collèges. Le Frère Joseph Pequignot, de la Compagnie de Jésus, trésorier-adjoint de l'Université de St. Louis, m'a dit qu'il n'avait jamais eu un tel sentiment de son importance que le jour où il porta à la banque un chèque d'un million, part de l'Université dans ce don énorme. Cependant, la somme ne couvrait que la moitié des traitements annuels des enseignants. D'une façon générale, l'Université de St. Louis reçoit des grandes entreprises une moyenne annuelle de $\$ 175,000$.

\section{Le sens de la responsabilité sociale}

Ce sens tout nouveau du rôle social du patronat, le fait que les dirigeants des grandes entreprises acceptent de plus en plus leurs responsabilités sociales constituent autant de freins à l'utilisation brutale de la puissance économique. De nos jours, cette attitude est systématiquement inculquée aux élèves de nos quelque 600 écoles de Hautes Etudes Commerciales. Cette «professionalisation 》 des fonctions de dirigeants fut parfaitement formulée par le Dr. James B. Conant, doyen de l'Université de Harvard:

- Plus que jamais, il nous faut à la tête des grandes entrepriges des hommes capables de pleinement apprécier leurs responsabilités, envers leur firme comme envers cette remarquable société d'hommes 
libres qui a prospéré dans notre continent. Ces hommes doivent comprendre, en plus de leur problèmes professionnels, le climat économique et social dans lequel ils travaillent; leur formation ne doit pas être inférieure à celle de nos avocats et médecins».

Il suffit de parcourir les revues professionnelles que lisent les chefs d'entreprise, comme Fortune, The Harvard Business Review ou Business Weeks, pour se rendre compte qu'on souligne de plus en plus les implications morales du processus économique. ${ }^{45}$ C'est sans doute aussi ce sens de la responsabilité sociale qui est à la base du Code de Conduite Morale esquissé par la centrale syndicale AFL-CIO, et du Code de Conduite Morale publié plus récemment par l'Association Nationale des Industriels. Au printemps dernier, comme on allait arriver à la date prévue pour la négociation d'un nouveau contrat collectif avec le syndicat des travailleurs de l'Automobile, $\overline{M r}$. George Romney, président de l'American Motors Company, prit une initiative révélatrice de cet état d'esprit: il invita à Détroit un petit nombre d'eoclésiastiques compétents, venant de toutes les parties du pays, et qui allaient conseiller la société sur les implications morales de la situation. De toute évidence, ces réunions ne correspondaient point à un but publicitaire. D’après le Père Léo C. Brown, de la Compagnie de Jésus, qui participa à la conférence, la société fit preuve d'une sincérité totale: elle apporta sa comptabilité, communiqua les estimations des ventes à venir, révéla sa position financière, admit ses faiblesses, de manière à confronter ces éléments avec les demandes déjà rendues publiques du syndicat.

En somme, il apparaît, - c'est du moins la thèse de l'auteur de cet article, - que les nombreux \&contrepoids» intervenant dans la société industrielle moderne se substituent, de façon satisfaisante, au régime d'une propriété privée largement répartie en ce qui concerne la protection des libertés de l'individu. Reste à savoir si ces contrepoids peuvent également se substituer à la seconde fonction de la propriété privée, c'est-à-dire à la garantie de la sécurité matérielle?

(45) Parmi les ouvrages étudiant ce problème, citons: Howard R. Bowen, Social Responsabilities of the Businessman, Harper, New-York, 1953 (Troisième d'une série de 12 volumes sur la morale et l'économie de la Société, publiée, par un comité du Conseil National des Eglises, avec l'appui de la Fondation Rockefeller); Francis Sutton, Seymour E. Harris, Carl Kaysen, The American Business Creed, Harvard Press, Cambridge, 1956, et Sylvia et Benjamin Selekman, Power and Morality in a Business Community, McGraw-Hill, New-York, 1958. 
Comme nous savons, il existe en Amérique une masse prodigieuse de richesses appartenant à des particuliers, sous forme de maisons, objets mobiliers, comptes d'épargne. Or, bien que l'on compte environ 8 millions d'entreprises individuelles, ${ }^{48}$ l'Américain «moyen est un salarié. Nous sommes fiers de savoir que l'épargne personnelle atteint un niveau constant de 484 milliards de dollars, plus 280 milliards de valeurs mobilières détenues par des particuliers, mais le fait demeure que l'existence de 82 Américains sur 100 dépend du bulletin de paye hebdomadaire ou mensuel. Situation peu satisfaisante pour ceux dont l'idéal reste le chef de famille établi à son compte et assurant lui-même la sécurité matérielle des siens, travaillant selon sa volonté, avec son propre outillage, - bref, un régime ou chacun gagne sa vie en blanchissant le linge d'un voisin.

\section{La sécurité économique ?}

La sécurité économique ou matérielle est-elle possible dans une société industrialisée? Une sécurité comparable à celle qu'imaginent les «Distributionnistes», basée sur la possession d'une terre ou de moyens de production? Ou bien, existe-t-il des forces d'équilibre, des contrepoids, capables d'assurer une protection efficace contre la misère de la vieillesse et contre celle, tout aussi affreuse, des périodes de crise économique?

A mesure que l'économie se développe, le volume des richesses s'accroît. Grâce aux contrepoids qui fonctionnent partout dans l'univers occidental, ces richesses sont distribuées de plus en plus équitablement. On trouve, certes, aux Etats-Unis des familles dont le niveau de vie (comparé au standard américain) a subi une nette déduction: surtout dans les fermes «marginales», les régions en proie aux crises chroniques, chez les ouvriers agricoles ambulants, dans certaines catégories d'âge, ou encore là où le chef de famille, pour telle ou telle raison, est incapable de travailler à temps entier. Cependant, il est établi que le pourcentage des familles vivant d'un revenu réduit est à peine la moitié de ce qu'il fut en 1935. En 1929, donc dans un passé très récent, seul le quart des familles américaines disposait de $\$ 4,000$. par an (impôts déduits, et calculé en dollars de 1956). Aujourd'hui, c'est le cas de trois familles sur quatre, - ce qui représente 31 millions de familles.

(46) Ce chiffre comprend les $4,800,000$ fermes figurant au recensement de 1954 . 
Les Talmudistes marxistes s'en arrachent peut-être les cheveux, mais ils ne peuvent rien contre le fait qu'aujourd'hui, le revenu moyen en Amérique est onze fois plus élevé que 75 ans plus tôt. ${ }^{47}$ Depuis 1919 , le revenu familial a triplé. Le gâteau à partager n'a pas seulement augmenté de volume; on le distribue aussi beaucoup plus équitablement.

Depuis le fameux Employment Act de 1946, le gouvernement fédéral est tenu à surveiller le bon fonctionnement de l'économie américaine, et de prendre des mesures susceptibles de prévenir des fluctuations extrêmes qui mettraient en péril la sécurité matérielle des individus. D'après ce texte, le président doit adresser au Congrès, au début de chaque année, un rapport sur la situation économique. Les chiffres cités dans le rapport de 1957 démontrent que la sécurité matérielle peut exister dans un régime d'expansion. Le revenu national, impôts déduits, était de 286,6 milliards de dollars, dont $7.3 \%$ furent versés à l'épargne. Le salaire hebdomadaire de l'ouvrier d'usine est de 81 dollars. ${ }^{48}$

Ce revenu personnel est étayé par le régime des retraites, garantie d'une sécurité matérielle pour la vieillesse, et soutient précieux en période de chômage. L'année dernière, la contribution gouvernementale aux fonds de retraite s'élevait à 20 milliards de dollars. Au cours des dix dernières années, les versements privés ont augmenté de 20 fois. L'allocation-chômage est, en moyenne d'un peu plus de $\$ 30$. par semaine; l'Etat le plus avare verse $\$ 20$. le plus généreux $\$ 45$. Dans certaines industries de base, - voitures, verre, acier, - les employeurs complètent les allocations officielles, versant aux chômeurs jusqu'à $65 \%$ du salaire, et cela pendant 39 semaines. ${ }^{49}$ Il existe également un programme fédéral d'assistance aux aveugles, aux mères indigentes, etc. En 1956, 106 millions de chefs de famille étaient titulaires d'une assurance-vie, d'un capital moyen de $\$ 7,500$.

En présence de ces chiffres, il faut se demander: ce vaste ensemble de placements, allocations, retraites donne-t-il les mêmes garanties

(47) Economic Growth in the United State, ouvrage cité, pp. 12 et 38 . La part du revenu national attribuée aux petits salariés, selon le Professeur Slichter, s'accroit d'une façon fulgurente. Ouvrage cité p. 195.

(48) Secrétaire d'Etat au Travail, James P. Mitchell, à la Conférence de l'ILO, le 6 juin 1958.

(49) Voir Franc's Corrigan, The Big Gaw Debate, Social Order, avril 1955 et Joseph Becker, Social Order, juin 1955. 
de sécurité matérielle qu’un régime de propriété privée largement répartie, tel que l'envisage la pensée sociale du catholicisme?

Une phrase passablement obscure de Messmer suggère que notre conception traditionnelle de la propriété s'avère peut-être trop étroite, aujourd'hui:

- Dans la mesure du possible, tout individu devrait posséder les biens nécessaires à l'accomplissement de sa tâche personnelle et sociale, ou encore, il devrait être en mesure, grâce au régime social, de les acquérir sous forme de rétribution de son travail $» .{ }^{\circ 0}$

\section{Une extention de la notion de propriété}

Au fond, pourquoi la notion de propriété ne comprendrait-elle pas les valeurs économiques d'ancienneté dans l'emploi, les droits acquis (et croissants) à la retraite, même si ces éléments ne sont pas légalement attachés à l'ouvrier? D'ailleurs, on considère de plus que la retraite appartient au travailleur, puisqu'elle lui a été accordée à la place d'un supplément de salaire. Il s'agit donc bien d'une forme de propriété. Par conséquent, le travailleur qui change d'emploi devrait être autorisé à emporter son droit à la retraite, avec ses versements accumulés.

En 1957, l'évêque portugais Ferreria Gomèz, à l'occasion de la Semaine d'Etudes Rurales Portugaise, déplora cette disposition du Code Napoléon qui impose le partage égal des biens du défunt entre ses enfants. Une grande partie des terres du Portugal a été si bien partagee qu'aujourd'hui, on ne peut plus les travailler avec profit. Sur une terre économiquement non viable, le droit de propriété apparait comme une mauvaise plaisanterie. Mais il est tout aussi absurde d'interdire l'exercice de sa profession à un homme parce que, dans la ville d'où il vient, son métier appris était considéré comme tombant sous la juridiction du syndicat de la photo-gravure, alors que dans la ville où il arrive, le même métier dépend du syndicat des lithographes. Or, nous connaissons tous les droits de propriété attachés aux marques commerciales et aux brevets. Nous admettons que la Cour Suprême, dans Pintérêt commun, limite les droits de propriété rurale en interdisant aux exploitants d'abattre des arbres de forêts sans en avoir

(50) Ouvrage cité, p. 796. 
planté d'autres. Ne devrait-on établir un parallèle avec les droits d'ancienneté d'un groupe d'ouvriers?

Peut-être, en réfléchissant bien, arriverons-nous à constater qu'il existe en Amérique beaucoup plus de propriété privée, - et fort bien répartie - que nous ne le pensions. ${ }^{51}$

\section{La fonction du développement humain}

Reste la troisième fonction de la propriété privée: le développement de la personne humaine. Est-ce que, dans ce domaine, les forces d'équilibre pourront également se substituer à notre régime idéal?

Nous allons rencontrer ici le problème soulevé par Walter Reuther, celui de la survie de l'individu exposé à la pression des géants, - le Grand Gouvernement, les Grandes Entreprises, les Grands Syndicats. Problème qui préoccupait le défunt pape Pie XII, notamment dans ses messages de Noël de 1952 et 1957, lorsque Sa Sainteté, après avoir salué les magnifiques résultats obtenus par la société industrielle, mit les fidèles en garde contre certains aspects excessivement mécanisés de la vie moderne.

Il y a quelques semaines, le Père William J. Smith, de la Compagnie de Jésus, posa la question suivante:

«Le système capitaliste façonne-t-il indûment l'existence de ses dirigeants et, en même temps, de ceux qui travaillent comme salariés dans son cadre? Et dans quelle mesure l'individu moyen subit-il servilement les mille pressions publicitaires sans lesquelles, nous diton, le régime ne saurait exister?»

Questions également traitées par deux ouvrages, L'homme de lorganisation et The Hidden Persuaders. Le premier affirme qu'une civilisation commerciale, avec son culte du succès matériel, aboutit au conformisme et à la perte de toute personnalité. Le second déclare que nos craintes inconscientes, nos complexes, nos désirs sont constamment maniés et modelés par des entreprises recherchant uniquement leur profit financier.

(51) De toute évidence, les récentes suggestions de Kelso et Adler dans le Manifeste Capitaliste augmenteraient simplement le nombre des détenteurs de titres - symbole assez maigre de la propriété privée. 
Affirmations difficiles à établir ou à réfuter, puisqu'elles sont basées sur une analyse de tendances. Cependant, le grand succès remporté par ces deux livres contient peut-être l'admission implicite du bien-fondé de leurs thèses, - ou encore, une capacité étonnante et réjouissante d'auto-critique. Toutefois, les remèdes seraient d'ordre spirituel et pédagogique plutôt que de nature économique. Ce qui ressort d'un avertissement que le Père John F. Cronin formule dans son ouvrage Social Principle and Economic Life:

«Nous semblons avoir perdu la faculté de distinguer entre les moyens et la fin, en faisant de la chasse aux biens matériels, souvent superflus, une fin en soi. Nous perdons de vue l'individu et la famille, la propriété privée qui en est la base économique, nous oublions les valeurs réelles des tâches sociales et économiques ». ${ }^{52}$

L'aspect le plus inquiétant peut-être de la société actuelle est linvraisemblable prix qu'il faut payer pour faire entendre \& un autre son de cloche». L'ancien sénateur Moody, après avoir étudié la possibilité de créer un journal d'opposition à Détroit, arriva à l'estimation suivante: le démarrage d'un journal digne de ce nom dans une grande ville exigeait un capital initial de 16 millions de dollars!

\section{Des mythes romantiques}

De toute manière, n'affirmons pas trop vite que l'emploi généralisé de la machine s'oppose au développement, à l'épanouissement de l'homme. Il est certes courant, - et même bien vu - d'opposer l'honnête artisan, fier de ses capacités professionnelles, à l'ouvrier abruti, pris dans les rets de la machine inhumaine, comme Charlie Chaplin dans les temps modernes. On parle aussi beaucoup de la belle spiritualité des paysans de Millet. Pour ma part, cependant, je suis incapable de reconnaître, dans les ouvriers américains que je connais, - dont plusieurs pères de mes compagnons Jésuites, - le type du prolétaire brutalisé, malmené, dépersonnifié. A mesure que les procédés de fabrication deviennent plus complexes, les connaissances requises se développent et se spécialisent.

En 1957, près de $50 \%$ des ouvriers de l'industrie privée avaient à leur actif au moins quatre années de collège. Ils avaient donc

(52) Publié récemment chez Bruce, Milwaukee. 
poursuivi leur études jusqu'à l'âge de 19 ans. Entre 1940 et 1957, le nombre des ouvriers ayant quatre années de collège et davantage a presque doublé. ${ }^{53}$

Au début de l'année 1958, un rapport gouvernemental indiquait que la durée de la fréquentation scolaire de louvrier \&moyen » américain était de 11.8 ans. Ce qui donne un tableau d'où le primaire semble absent. Ceux qui existent encore se recrutent surtout parmi les Noirs et les frontaliers mexicains, terrassiers ou moissonneurs ambulants. D'ailleurs, toutes les grosses entreprises font un énorme effort pour permettre à leurs ouvriers de se perfectionner davantage. Le programme de formation professionnelle de la General Electric dispose d'un budget annuel de 40 millions de dollars.

On observe dans l'industrie américaine un refus total du système européen où une classe est censée fournir le muscle, l'autre (et uniquement l'autre) le cerveau. De plus en plus, nos entreprises aherchent leurs cerveaux parmi leurs ouvriers.

L'esprit de création s'émousse-t-il vraiment lorsqu'on doit travailler pour un patron? L'extraordinaire développement de la fabrication des ensembles «Faites-le vous-mêmes» semble démontrer le contraire. Il paraît, certes, que les distractions offertes à des milliers de personnes entraînent un conformisme de goûts et d'opinions; en revanche, les millions de télespectateurs qui ont vu - une seule fois, - Hamlet sur le petit écran sont probablement plus nombreux que tous les fervents du théâtre qui ont assisté aux représentations, depuis la mort de Shakespeare, au XVIe siècle.

Manifestement, les possibilités d'épanouissement dépendent dans une certaine mesure des loisirs. En l'espace de 25 ans, l'ouvrier américain a gagné de 15 à 20 heures de liberté par semaine. Sait-il les mettre à profit? De toute manière, il aura plus de temps à consacrer à sa famille. J'aimerais citer à ce propos l'aneadote qui me fut rapportée par un ami instituteur. Un samedi matin, cet ami, occupé à corriger des copies, entendit du bruit sous ses fenêtres. Il regarda dehors: dans la cour, un voisin, ouvrier qualifié, jouait au ballon avec ses fils. Mon ami, avec un sourire mélancolique, se rappela son propre père, également ouvrier qualifié, - mais qui, rentrant d'une journée interminable à l'usine, était toujours si fatigué qu'il passait le di-

(53) The Economy of the American People, ouvrage cité, p. 36. 
manche, sa seule journée de repos, à dormir, et que les enfants devaient marcher sur la pointe des pieds pour ne pas le réveiller.

\section{Conclusion}

Le présent exposé (destiné en principe à un public européen) ne doit pas constituer un panégyrique du système américain. Il pose simplement le problème: notre vieille conception de la propriété, - de petites unités de production, petites parce qu'il faut qu'elles soient petites, - reste-t-elle viable aujourd'hui, compte tenu de l'accroissement de la population mondiale qui ne peut être nourrie que par un accroissement massif des richesses produites? But qui ne peut Atre atteint que par la production en série, laquelle ne peut être obtenue que par l'emploi toujours plus généralisé de machines, emploi qui ne peut être obtenu (malheureusement, peut-être) que par la concentration de la puissance économique et de la main-d'oeuvre qualifiée. L'économie américaine d'aujourd'hui ne correspond guère aux espoirs et principes de la pensée catholique qui tend à la large répartition d'une propriété réelle. Cependant, c'est un système dans lequel les libertés individuelles sont suffisamment protégées, la sécurité matérielle à peu près assurée, et qui offre au moins uns chance d'épanouissement personnel. Il n'y a pas de pauvres qui viennent mendier un morceau de pain à la porte de nos établissements religieux.

Un observateur critique de la vie américaine, le professeur Victor Ferkiss, a très bien formulé le problème:

< Etant donné que nos difficultés sont d'ordre social plutôt qu'économique, les réformes doivent s'attaquer non à la structure économique mais à l'ensemble de nos relations sociales. Il s'agit donc non de réorganiser les systèmes de distribution ou les contrats collectifs, mais notre genre de vie en commun, et, surtout, de veiller à élever le niveau de nos aspirations intellectuelles, esthétiques et spirituelles $\gg .{ }^{54}$

\section{Des buts trompeurs}

Aucun Américain intelligent ne contestera que notre système économique comporte des lacunes. Nous employons aujourd'hui 11.5 millions de femmes mariées, $-29 \%$ des épouses. On estime à 3

(54) Social Action in the Affluent Society, Social Order, septembre 1958. 
millions le nombre des hommes qui oocupent deux emplois. Dans quelle mesure ces choses-là sont-elles inévitables? Pour ma part, je n'en sais rien. En mai 1957, SOCIAL ORDER, dans un article intitulé «La Conscience Chrétienne et l'Economie de l'Abondance» se demandait si notre prospérité n'était pas le résultat d'une sorte de supercherie puisque l'industrie et le commerce vendraient aux consommateurs des produits dont ceux-ci n’avaient pas vraiment besoin. Les résultats de notre enquête furent stupéfiants. Nous découvrîmes que l'une des branches les plus actives de l'époque était - les salons de beauté spécialisés dans les traitements amaigrissants. Une commission du Congrès a pu constater que le public américain dépensait annuellement quelque 100 millions de dollars en produits amigrissants, appareils, bains etc. - une fraude colossale, puisque le seul moyen connu de maigrir est le régime alimentaire. Dans cet ordre d'idées, je me souviens d'une annonce vantant une pâtée spéciale pour chiens d'appartements qu'une existence trop sédentaire avait rendus obèses. D'un autre côté, l'ouvrage THE AFFLUENT SOCIETY, dans lequel le professeur Galbraith, de Harvard, stigmatise labandon des valeurs culturelles à la suite de la chasse au profit et à la productivité, n'eut aucun mal à devenir un best seller.

\section{Un système incohérent?}

Nous vivons dans un système d'économie mixte où le pouvoir de décision est décentralisé. L'un des inconvénients majeurs de cette méthode fut révélé par Roger M. Blough, président de la United Steel, alors qu'il déposait devant une commission d'enquête au sujet des prix pratiqués dans sa branche. Mr. Blough rappela qu'en 1948, sa société, ayant baissé le prix de l'acier de $\$ 1.25$ la tonne, refusa d'augmenter les salaires. C'était, expliquait-on au syndicat, un effort pour freiner l'inflation. Le résultat?

«Nous aurions pu essayer tout aussi bien d'arrêter un train avec une fronde. Trois mois plus tard, nous dûmes renoncer à peser sur le prix de l'acier, accorder une augmentation de salaire et trouver un moyen de remettre de l'ordre dans nos affaires. A présent, nous avons compris: aucune entreprise, aucun syndicat ne saurait à lui seul freiner l'inflation ».

On découvre ainsi qu'il n'existe aucun mécanisme permettant aux chefs d'entreprise et aux syndicats de se consulter, et encore moins de 
coordonner leurs efforts dans l'intérêt de l'économie nationale. D'ailleurs, toute tentative dans ce sens exposerait ses auteurs aux foudres de la législation anti-trust, ainsi qu'aux soupçons d'une opinion publique toujours prête à flairer une collusion aux dépens du consommateur.

La pensée catholique estimera, sans doute, qu'une telle situation frise l'incohérence et l'irresponsabilité. Elle aura tendance à attribuer la récente récession à l'absence de tout planning généralisé. ${ }^{55}$ Notre économie a nettement démontré qu'elle n'échappe pas aux influences cycliques; d'ailleurs, comme l'homme dépense et économise selon ses caprices du moment, elle ne leur échappera jamais. Je pense, cependant, que nous disposons de «stabilisateurs 》 bien implantés qui amortiront les chocs des crises.

\section{Ne défions pas les faits}

La pensée sociale du catholicisme n'a rien à gagner a ressasser les positions prises et reprises dans les manuels. Ces doctes ouvrages décrivent une situation qui, probablement, n'a jamais existé et qui, de toute manière, ne pourra jamais être créée. Soyons sérieux: il s'agit de critiquer, sincèrement et intelligemment, un système qui permet à 3 millions de personnes de fréquenter collèges et universités, à $98 \%$ des couples mariés d'avoir un logement à eux, de financer la défense du monde libre contre l'impérialisme communiste et d'aider les pays sous-développés, - et, avec tout cela, d'élever constamment notre niveau de vie.

Quoi qu'il en soit, les jeunes catholiques américains qui achètent des maisons à tempérament, payables en 30 ans, qui mettent au monde des enfants à un rythme alarmant pour nos écoles paroissiales, ces jeunes catholiques ne paraissent pas trop mécontents de ce système. Mieux encore, ils lui font confiance pour l'avenir.

(55) En septembre 1958, le chiffre des chômeurs était de 4.1 millions sur un total de 68.7 millions de salariés. Le chômage affectait surtout certains secteurs do l'industrie lourde. 